\title{
Linx
}

Revue des linguistes de l'université Paris X Nanterre

$50 \mid 2004$

Variation sémantique et syntaxique des unités lexicales : étude de six verbes français

\section{Le verbe paraître : surgissement, manifestation, impression, ouï-dire}

\section{François Thuillier}

\section{(2) OpenEdition}

\section{Journals}

Édition électronique

URL : http://journals.openedition.org/linx/131

DOI : 10.4000/linx.131

ISSN : 2118-9692

Éditeur

Presses universitaires de Paris Nanterre

Édition imprimée

Date de publication : 1 juin 2004

Pagination : 15-32

ISSN : 0246-8743

Référence électronique

François Thuillier, «Le verbe paraître : surgissement, manifestation, impression, ouï-dire », Linx [En ligne], 50 | 2004, mis en ligne le 27 janvier 2011, consulté le 30 avril 2019. URL : http:// journals.openedition.org/linx/131; DOI : 10.4000/linx.131 


\section{Le verbe parâ̂tre : surgissement, manifestation, impression, ouï-dire}

\section{François Thuillier \\ UMR 7110 (Université Paris 7)}

La plupart des travaux linguistiques proposant des éléments de réflexion sur le verbe paraître abordent l'étude du verbe dans le cadre de la description d'un champ de valeurs spécifique. Paraître est ainsi pris en compte d'une part comme verbe de modalité épistémique, et d'autre part comme verbe "médiatif», c'est-à-dire permettant, selon la formule de Z. Guentchéva et al., «de marquer l'attitude de distanciation et de non-engagement que manifeste l'énonciateur à l'égard des faits qu'il présente $»^{2}$. Il n'est pas question pour nous de nier l'apport de ces travaux, mais de souligner que la perspective qu'ils adoptent a pour effet de morceler l'identité de paraître. En n'abordant l'étude du verbe que dans le cadre d'un champ notionnel, elles transforment les différentes acceptions de paraître en autant de verbes homonymes. En outre, l'étude de paraître dans le cadre de la description d'un champ notionnel revient à situer le travail d'analyse en un point où le sens serait déjà constitué. Les descriptions de paraître dans le cadre de l'étude de la modalité épistémique, ou de la catégorie de l'«évidentialité » présentent en effet la caractéristique commune de poser, dès le départ, le verbe comme verbe modal ou évidentiel, et ne peuvent entreprendre de déterminer ce qui le rend à même d'engendrer ce type de valeurs.

Nous considérons au contraire que seule une caractérisation unitaire de l'unité, formulant ce qui constitue la contribution régulière du verbe aux énoncés, permet de retracer les mécanismes de création des valeurs constatées, c'est-à-dire, par exemple, de déterminer comment paraître rend possible un effet de modalisation de l'énoncé attributif dans lequel il apparait. Le propos de cet article sera ainsi de montrer que la diversité des valeurs associables aux énoncés dans lesquels figure le verbe paraître peut être décrite en fonction d'un schéma abstrait unique, dont on postule que le verbe est

\footnotetext{
${ }^{1}$ Merci à Paul Athanassiadis, ainsi qu'aux contributeurs du présent numéro de Linx. Je remercie également les relecteurs anonymes de la revue pour leurs remarques et suggestions.

${ }^{2}$ Z. Guentchéva et al. (1994: 139).
} 
la trace matérielle. Il s'agira de formuler une hypothèse unitaire permettant de circonscrire ce qui, de la diversité des effets de sens qui lui sont associés, ressortit en propre à ce verbe ${ }^{3}$.

Après une brève revue de travaux consacrés à paraître et, plus généralement, aux verbes renvoyant à une problématique de la perception, on formulera une hypothèse de caractérisation générale du verbe paraitre, que l'on mettra en œuvre dans la description de quatre acceptions du verbe. Ces différentes acceptions, représentatives de la diversité des valeurs associées au verbe, présentent en outre l'intérêt de relever de l'une ou l'autre des quatre constructions syntaxiques qu'admet paraitre. Seront ainsi pris en compte 1) les emplois intransitifs du type Le soleil parut, qui marquent l'arrivée, l'entrée, le surgissement d'un élément en un lieu ; 2) les emplois causatifs tels que $I l$ ne laissa rien paraitre de son chagrin, qui renvoient à la manifestation de quelque chose, a priori soustrait à la vue ; 3) les emplois attributifs de la forme Cela lui parut bon, qui renvoient à un jugement d'un sujet relativement à un état de choses; et 4) les emplois impersonnels tels que Il paraît qu'il est venu, qui indiquent qu'un fait est rapporté, porté à la connaissance de quelqu'un.

\section{Quelques remarques sur une approche mentaliste de l'étude de paraître}

L'élucidation de ce qui fonde l'unicité d'un verbe tel que paraître, par-delà la diversité de ses emplois, peut être envisagée selon différentes approches. Nous proposons d'examiner dans cette section une démarche selon laquelle la définition du lexème est donnée comme la transcription en langue d'une image mentale. L'étude de P. Ouellet et al. intitulée La représentation des actes de perception : le cas de "paraitre ", se fonde sur le postulat d'un isomorphisme entre le fonctionnement des langues et les processus perceptifs dont découlent nos représentations. Les auteurs posent en effet qu'il existe une relation étroite entre les structures morpho-lexicales et morphosyntaxiques des langues naturelles et l'activité sensori-motrice des sujets, qui, selon les auteurs, "contraint ou détermine une bonne part des structures topologiques et gestaltiques de notre "imagerie mentale" »". En conséquence, les auteurs considèrent que l'identification du contenu sémantique d'un lexème tel que paraître, doit nécessairement prendre en compte les dimensions phénoménologiques d'un acte de perception. Cette vision des «verbes de perception» comme reflets de l'activité humaine de perception est partagée par Ch. Dupas, qui, dans son livre Perception et langage : étude linguistique du fonctionnement des verbes de perception auditive et visuelle en anglais et en français, indique que son intention de découvrir un modèle du fonctionnement des verbes de perception auditive et visuelle «est habitée par le projet d'établir une

\footnotetext{
${ }^{3} \mathrm{La}$ formulation proposée ici entend donc circonscrire ce qui constitue l'apport à la fois spécifique et régulier du verbe à la construction de la valeur des énoncés. Cette caractérisation permettra d'envisager, dans une étape ultérieure, une étude des facteurs de variation, réguliers ou contingents, rendant compte de la diversité des modes de donation du verbe.

${ }^{4}$ P. Ouellet, et al. (1994 : 136).
} 
corrélation entre les différents fonctionnements linguistiques et les différentes modalités de l'expérience perceptive $»^{5}$. Le modèle qu'élabore l'auteur a ainsi pour but de mimer, à l'intérieur des opérations de langage, les opérations perceptives, la structure du fonctionnement des verbes de perception étant vue comme un reflet parfait de la structure des actes perceptifs ${ }^{6}$. Cette vision de la langue comme icône, ou comme reflet, amène les auteurs à poser un rapport de motivation de la langue par l'extralinguistique. Ainsi pour P. Ouellet et al., le « verbe paraitre en français garde (...) la trace, dans sa structure lexico-sémantique, d'une complexité phénoménologique de l'acte même de paraitre (...) $)^{7}$. Selon les auteurs, ce sont des contraintes perceptives qui régissent non seulement l'aperception du sens ou de l'« image mentale » de paraitre, mais aussi la formation même des lexèmes qui les symbolisent, ceux-ci reproduisant «iconiquement» l'acte auquel ils renvoient ${ }^{8}$.

Si la pratique d'intégration de faits extralinguistiques dans l'analyse des phénomènes linguistiques peut apparaitre comme une évidence ${ }^{9}$, elle se heurte cependant à un certain nombre de difficultés. Outre le fait qu'il est difficile, dans une optique mentaliste, de valider ou d'invalider les hypothèses concernant des entités qui, parce que mentales, sont par définition inaccessibles, le problème essentiel que posent les définitions fondées sur un rapport de motivation extralinguistique des unités lexicales est qu'elles font fréquemment intervenir des critères de plausibilité mondaine, qui sont nécessairement subjectifs, et directement liés aux représentations du linguiste ${ }^{10}$. Pour ne prendre qu'un exemple, $\mathrm{Cl}$. Vandeloise pose un contraste entre les énoncés suivants :

\footnotetext{
${ }^{5}$ Ch. Dupas (1997: 7).

${ }^{6}$ Idem, p. 339.

${ }^{7}$ Idem, p. 154. Cl. Vandeloise (1990 : 159) énonce particulièrement clairement cette thèse de la motivation des formes linguistiques par le monde, dans le cadre d'une étude sur la préposition sur: «la relation $\mathrm{P}$ (orteur)/p(orté) est une relation du monde extralinguistique qui motive l'usage de la préposition sur. Elle se manifeste par les mêmes caractéristiques dans le monde entier, et toutes les langues associent probablement une forme linguistique à l'ensemble de ses caractéristiques perçues globalement ».

${ }^{8}$ P. Ouellet et al., p. 150.

${ }^{9}$ Cette option théorique est très largement dominante, et même parfois présentée comme unanime. F. Gayral (1998 : 74) déclare ainsi que «le présupposé [de l'indépendance du sens par rapport à des connaissances extralinguistiques] parait beaucoup trop violent puisque tout le monde admet l'intervention de diverses connaissances dans la définition du sens. (...) La difficulté de ce cloisonnement s'illustre parfaitement en sémantique lexicale où il est plus difficile d'éviter les rapports étroits qu'entretiennent la langue avec le monde et d'évincer dans la définition du sens d'un mot le fait qu'il évoque nécessairement une expérience, un comportement standardisé, qu’il nous permet d'accéder à tout un ensemble d'informations qui lui sont intimement associées ».

${ }^{10}$ Cf. C. J. Fillmore (1970 : 56) : «La définition mentaliste n'est d'aucun secours à quiconque veut savoir s'il comprend une forme linguistique, pour la seule raison qu'il n'y a aucun moyen de savoir si les images qu'il a dans l'esprit quand il produit ou rencontre la forme sont les mêmes que celles de ses interlocuteurs ».
} 


\section{[a.] L'oisean est à l'extérieur de la cage.}

[b.] ?Le couteau est à l'extérieur de la cage.

et précise : «par ces phrases et ces dessins, je veux montrer que l'objet de la relation à l'extérieur doit être pragmatiquement lié à son sujet. Ainsi la phrase [b] est-elle moins acceptable que la phrase [a], parce que les couteaux ont moins de relation avec les cages que les oiseaux. C'est du moins ce qui résulte de notre connaissance du monde normal. Ceci n'exclut pas l'existence possible d'un vieil excentrique qui aime mettre les oiseaux dans des tiroirs et les couteaux dans des cages. Si la cage ci-dessous était la sienne, le jugement devrait être inversé. Quelle que soit l'étendue de l'imagination du lecteur, je le prierai cependant de bien vouloir m'épargner ce type d'interprétations ${ }^{11}$. On voit donc que le degré d'acceptabilité des phrases est posé comme étant fonction de la plus ou moins grande imagination du lecteur. Étant donné le caractère subjectif, et donc non répétable, d'un jugement concernant la plausibilité d'un événement extralinguistique, divers auteurs ont insisté, au contraire, sur la nécessité de conserver une différence nette entre la plausibilité d'un énoncé et sa grammaticalité ${ }^{12}$.

Par ailleurs, dans une optique où les formes linguistiques sont vues comme des transcriptions d'images mentales ou d'une activité de conceptualisation du monde, le risque est grand de voir la linguistique devenir une discipline dépendante d'autres disciplines, telles que la psychologie. L'objet d'étude se trouve, de fait, déplacé dans de nombreux travaux d'obédience cognitiviste: dans cette perspective il s'agit souvent moins, pour le linguiste, de collecter et d'interpréter les phénomènes formels livrés par l'observation d'une langue, que de scruter le fonctionnement de l'esprit du sujetlocuteur. P. Ouellet pose ainsi que les processus de signification « ont bien sûr à voir » avec les contraintes formelles des langues naturelles, mais qu'ils s'appuient d'abord sur le fonctionnement de la conscience des sujets ${ }^{13}$. On mesure le risque de réduction de l'élaboration métalinguistique à une description hypothétique des mécanismes mentaux, dans les travaux qui, plus ou moins explicitement, posent une assimilation entre les données linguistiques et les faits psychologiques. Ch. Dupas considère ainsi que dans les cas où l'activité de « voir » ne présente pas de différence avec l'activité de « regarder», les verbes voir et regarder sont synonymes ${ }^{14}$. On trouve une approche

${ }^{11} \mathrm{Cl}$. Vandeloise (1986: 61-62).

${ }^{12}$ Cf. H. A. Gleason (1961 : 198), qui souligne la différence entre une phrase «nonsensique » et une phrase agrammaticale : «A non-sensical sentence may be quite grammatical. If so, this merely means that, should the occasion arise, the sentence in question might well be said, and in that situation might seem quite natural, even usual. A non-grammatical sentence cannot be used unless the language should change ». Voir également sur ce point A. Daladier (1990).

13 P. Ouellet (1994: 137).

${ }^{14}$ L'auteur (1997 : 259-260) affirme ainsi que « si le marin peut voir arriver la tempête par un simple changement dans l'orientation des vents, si le pilote de planeur peut voir, lorsqu'il est en 'spirale ascendante', une invisible colonne d'air chaud qui le porte (...), si le musicien peut entendre les plus subtiles nuances dans l'interprétation d'un morceau, c'est parce que dans tous ces cas, le pouvoir de perception est le résultat d'une expérience, d'un apprentissage, et d'un affûtage des organes perceptifs ». Dans toutes ces occurrences, conclut Ch. Dupas, "il est évident que voir fonctionne 
similaire dans une étude de P. Ouellet sur d'autres verbes de perception, tels qu'observer ou apercevoir. La démarche mise en œuvre dans l'article consiste à inventorier de façon intuitive les actes extralinguistiques auxquels renvoient, dans certaines conditions, des mots tels qu'observer, contempler, etc. On donne ensuite à cette description le statut de représentation mentale, puis on assimile la signification du mot à cette représentation. Ainsi, P. Ouellet indique-t-il que l'on peut « difficilement apercevoir un objet pendant de très longues heures et l'observer pendant seulement une fraction de secondes : observer et examiner font voir plus en détail l'objet perçu et durent, par conséquent, plus longtemps qu'apercevoir ou entrevoir (...)». Puis il conclut: «il semble donc que la grandeur, de l'objet ou de l'acte même de perception, soit un paramètre essentiel pour la discrimination des différentes valeurs du lexique verbal renvoyant au champ perceptuel $»^{15}$.

On voit que ce type d'approches peut aboutir à donner à l'analyse spécifique des phénomènes langagiers un rôle secondaire, et à fonder l'étude d'un lexème sur des hypothèses concernant le comportement cognitif des locuteurs. La définition du verbe paraitre que proposent P. Ouellet et al. en fournit un bon exemple : constatant que, dans leur corpus, «il y a souvent un VOIR avant PARAÎTRE et que ce dernier est généralement suivi d'un COMME dénotant une interprétation ou d'un PENSER $»^{16}$, les auteurs font l'hypothèse que paraitre renvoie à une valeur intermédiaire sur le spectre qui va de l'extéro- à l'intéro-ception dans le champ complet de l'expérience perceptive. La définition proposée se fonde ainsi sur la conception que possèdent les auteurs des interactions des sujets avec la réalité mondaine: ce qui permet de considérer que paraitre est une valeur intermédiaire entre voir et penser répond à l'idée qu'un sujet commence par percevoir la réalité qui l'entoure, ce qui donne lieu dans un deuxième temps à une activité de pensée. Paraitre, dans cette perspective, nomme un processus occupant une position intermédiaire dans ce continuum d'actes mentaux.

\section{Hypothèse de caractérisation du verbe parâ̂tre}

La démarche que nous allons mettre en œuvre dans la suite de cet étude se distingue assez radicalement de celle que nous venons de présenter: dans la perspective adoptée ici, les données linguistiques correspondent à la fois à un point de départ de l'analyse et à un point d'arrivée, puisque les raisonnements métalinguistiques ont pour seul but d'éclairer le comportement sémantique et distributionnel de l'unité étudiée. La caractérisation du lexème prend la forme d'un schéma, articulant des paramètres, qui correspond à un principe dynamique d'interaction du verbe avec son environnement. Ce schéma peut ne pas avoir systématiquement de répondant lexical

comme regarder, car ce pouvoir voir ou ce pouvoir entendre est indissociable d'une attention, d'une réceptivité active et non d'une simple fonction d'appréhension'. Les italiques sont de l'auteur.

15 P. Ouellet (1992 : 179-180).

16 P. Ouellet et al. (1994: 151). 
dans l'énoncé ${ }^{17}$; cependant, dans le cas où ne figure textuellement qu'une partie des paramètres, il faut pouvoir en identifier une trace, sous forme de contraintes de cooccurrence de paraitre avec certains lexèmes, ou inversement, de tendances relevées sur les combinaisons de certains lexèmes avec paraître, ou enfin de contraintes interprétatives (par contrainte, on entend que l'interprétation doit être régulière).

Notre hypothèse, que la description des énoncés permettra d'expliciter, est que paraitre marque qu'une entité $\mathbf{X}$ est en elle-même hors d'atteinte, mais qu'elle se trouve appréhendée par un sujet de perception $\mathbf{S}$, par l'intermédiaire d'une autre entité (notée $\mathbf{X}^{\prime}$ ), seconde par rapport à $\mathbf{X}$.

Précisons que l'on entend par «second» la qualité d'une chose «qui dérive d'une chose première, primitive $»^{18}$; comme on le verra, ce caractère second de $\mathbf{X}$ ' se révèle dans le fait que $\mathbf{X}^{\prime}$ correspond, selon les cas, à une représentation de $\mathbf{X}$, un signe, un symptôme de $\mathbf{X}$, une impression de $\mathbf{X}$. Ce qui est perçu n'est donc pas $\mathbf{X}$ directement, mais $\mathbf{X}^{\prime}, \mathbf{X}$ restant en lui-même inaccessible. Cette formulation suppose qu'il existe un écart, une distance entre $\mathbf{X}$ et $\mathbf{X}$ ', cet écart pouvant être plus ou moins accentué, souligné, et donnant lieu à une gamme de mises en relation entre $\mathbf{X}$ et $\mathbf{X}^{\prime}$, d'où, comme on le verra, l'apparition dans les énoncés de problématiques de l'illusion, ou de la dissimulation de $\mathbf{X}$.

\title{
3. Analyse d'énoncés en fonction de cette hypothèse
}

\subsection{Description des énoncés du type Il parut entre deux gendarmes} tels que :

La propriété sémantique qu'il nous semble crucial de relever dans des énoncés

\author{
Soudain, il parait sur le seuil. \\ Le soleil parut enfin.
}

est qu'ils inscrivent les protagonistes dans une " mise en scène » d'un événement : ces énoncés renvoient à une entrée en scène d'un personnage, ou d'un objet. La problématique du «visuel», dont la pertinence est unanimement reconnue dans la définition de paraitre, est réelle, mais insuffisante à dire toute la spécificité de l'effet

\footnotetext{
${ }^{17}$ Comme l'indiquent C. Poirier, et al. (1998: 121-122) : «Si l'on observe des irrégularités apparentes dans différents emplois d'un verbe, ce n'est pas parce que ce schéma est bafoué, mais parce que nous projetons directement la structure syntaxique - la forme textuelle - sur la structure sémantique ». « Il existe donc une relative indépendance entre compléments sémantiques et textuels, un complément sémantique pouvant ne posséder aucune réalisation textuelle, ou au contraire en posséder une (totale ou partielle) dans un ou plusieurs compléments textuels ».

${ }^{18}$ Cf. Le Petit Robert.
} 
induit par paraître sur la valeur de l'énoncé ${ }^{19}$. Paraître induit une "théâtralisation » des événements, comme l'indique bien le Littré, qui glose le verbe dans l'énoncé :

Les mahométans parurent, conquirent et se divisèrent. (Montesquieu, cité par Littré)

par «venir sur le théâtre du monde». Pour étayer cette intuition d'une "théâtralisation" de l'événement auquel renvoie paraître, on dispose d'un ensemble d'arguments distributionnels, dont le plus évident est le nombre particulièrement élevé de co-occurrences du verbe avec le mot scène. Celui-ci représente en effet à lui seul $51 \%$ des $\mathbf{Y}$ dans les énoncés en $X$ paraît en $Y$, avec un $\mathbf{X}$ animé humain et un syntagme prépositionnel (désormais noté SP) s'interprétant comme un lieu (autrement dit, quand une personne paraît en un lieu, une fois sur deux, ce lieu est une scène $)^{20}$. D'autre part, dans les énoncés intransitifs de la forme $X$ paraît dans $Y$, avec un $\mathbf{X}$ animé humain, plus du tiers des lieux où $\mathbf{X}$ paraît correspond à des pièces de théâtre, ou des spectacles, comme dans :

Par exemple, ne voulut-il pas une fois me forcer à paraître dans Monsieur De Pourceangnac, à la scène des enfants? (P. Léautaud, In Memoriam, 1905)

alors que ce type de SP ne représente, pour surgir, se présenter, se montrer, apparaître, selon les cas, que de 0 à $2 \%$ des occurrences. On observe par ailleurs la récurrence du mot public en position de $\mathbf{Y}$, dans les énoncés de la forme $X$ paraît en $Y$ : public représente en effet à lui seul $40 \%$ de la classe des SP interprétables comme des circonstants de paraître. On a des énoncés du type :

La fiancée, ne devant pas paraître en public avant le jour du mariage, demeurait en baut dans les chambres de sa grand-mère, (...). (Z. Oldenbourg, Les cités charnelles ou l'bistoire de Roger de Montbrun, 1961)

Paraître est donc particulièrement compatible avec l'expression de la présence de spectateurs de l'événement. Inversement, si l'on indique explicitement dans l'énoncé que l'événement a lieu sans témoins, un énoncé avec paraître est bizarre :

?? Il parut dans la pièce sans que personne ne s'en aperçoive.

alors qu'on pourra avoir :

Il entra + arriva dans la pièce sans que personne ne s'en aperçoive.

On notera à ce propos que dans le corpus Frantext, tous les énoncés de la forme qqn voit paraître $X$ (soit 138 occurrences) sont à la forme affirmative ; il y aurait une contradiction à " ne pas voir paraittre $\mathbf{X}$ »; en revanche, on relève des énoncés tels que :

\footnotetext{
${ }^{19}$ Pour ne prendre que deux exemples, le TLF et Le Petit Robert classent ces emplois sous la rubrique « se présenter à la vue »; cf. également C. Guimier (1991 : 227), qui indique que dans les emplois du type, La porte s'ouvrit. Marie parut, «paraître évoque l'être ou le devenir visible du sujet ».

${ }^{20}$ On observe également une forte compatibilité de paraître avec un syntagme en sur scène. Les statistiques fournies dans cet article concernent l'ensemble des énoncés livrés par le corpus Frantext, de 1900 à nos jours.
} 
François Thuillier

Nous étions seuls, on ne m'avait pas vu venir et l'endroit était isolé. (L. Bloy, Exégèse des lieux communs, 1902)

où la substitution de paraître à venir donnerait un énoncé peu acceptable :

?? Nous étions seuls, on ne m'avait pas vu paraître et l'endroit était isolé.

De même, dans un énoncé tel que :

Le chat des Méricant surgit sous la table et sauta sur les genoux de Berthe. (J. Chardonne, L'Epithalame, 1921)

où le syntagme prépositionnel sous la table s'interprète comme quelque chose qui empêche de voir $\mathbf{X}$, on peut difficilement substituer paraître à surgir :

?? Le chat des Méricant parut sous la table et sauta sur les genoux de Berthe.

Cependant, ce dernier énoncé n'est pas strictement impossible. Pour le rendre acceptable, il faut fabriquer un contexte dans lequel sous la table ne constitue pas un obstacle à la perception de $\mathbf{X}$ (le chat), en imaginant par exemple que quelqu'un se trouve lui-même sous la table. Autrement dit, pour rendre l'énoncé acceptable, il faut imaginer que l'on place un spectateur dans une position lui permettant de percevoir l'arrivée de $\mathbf{X}$.

On a vu que la spécificité interprétative des énoncés avec paraître, est qu'ils renvoient à une «entrée en scène » d'un objet ou d'une personne. Une scène, comme l'indique T. Gallèpe, auteur de l'ouvrage Didascalies. Les mots de la mise en scène, possède deux caractéristiques principales. D’une part la radicale séparation entre deux «mondes », celui de la scène, et celui de la salle, des spectateurs ; et d'autre part le fait que la scène est un lieu où se déploie un monde représenté (une recréation d'événements), de telle manière qu'un public puisse assister à cette recréation ${ }^{21}$. On peut donc soutenir que dans les énoncés décrits ci-dessus, ce qui est perçu par une instance subjective (notée $\mathbf{S}$ ), correspond à une construction, une représentation (notée $\mathbf{X}^{\prime}$ ), produite à partir d'une personne ou d'un objet réel (noté $\mathbf{X}$ ), mais qui ne se confond pas avec lui, et qui est, par rapport à lui, seconde ${ }^{22}$.

${ }^{21}$ T. Gallèpe (1997 : 16). L'auteur cite A. Übersfeld (1982), qui souligne également ces deux caractéristiques : «Le comédien serait-il assis sur les genoux du spectateur qu'une rampe invisible, un courant à 100000 volts l'en séparerait encore radicalement. Même s'il y avait (comme dans le théâtre politique, le théâtre d'agitation) mise en scène d'un événement réel, ce réel aurait, une fois théâtralisé, un statut de non-réalité qui l'apparente au rêve ». Un rapide parcours des principaux emplois de scène le confirme : une scène, dans un film, est l'unité de temps spécifique de ce qui se déroule du point de vue du spectateur, et non du point de vue de l'organisation temporelle des événements du film (de même pour l'emploi «subdivision d'un acte", au théâtre, et contrairement à épisode). Faire une scène à quelqu'un, contrairement à faire une crise à quelqu'un, constitue aussi un spectacle, de même que filer une scène. La scène est faite à l'intention d'un public : ce qui était de l'ordre du privé est en fait ici un «show ». De même, dans une scène affreuse + touchante + de désolation + de carnage, les événements sont envisagés comme des spectacles.

${ }^{22}$ Le Petit Robert définit la représentation comme « le fait de rendre sensible (un objet absent ou un concept) au moyen d'une image, d'une figure, d'un signe ». Cf. également Diderot, dans le Paradoxe 
Cette formulation nous semble pouvoir rendre compte assez facilement de deux types d'occurrences de paraître, que l'on peut considérer comme des emplois prototypiques du verbe. On peut d'une part expliquer la valeur de ce que les grammaires et les dictionnaires nomment l'emploi absolu de paraître, dans lesquels le verbe est complément d'objet direct de verbes de visée ou d'intentionnalité, tels que falloir, s'occuper de, vouloir, chercher à, ou complément de noms tels que souci, besoin, rage, désir, comme dans :

Mais il savait qu'il trouverait à l'intérieur un public plus choisi, et en majorité féminin; donc mieux assorti à son propre désir de paraître, et à la couleur de ses pensées (...) (J. Romain, Les hommes de bonne volonté, 1932)

«Celui qui aime paraître » est une personne qui se crée un personnage, qui se met en scène (Le Petit Robert glose ces emplois par «se donner en spectacle »). L'interprétation péjorative provient du fait que celui qui aime paraittre fonde tout son mode d'être sur le personnage qu'il donne à voir $\left(\mathbf{X}^{\prime}\right)$, d'où une problématique de la dissimulation de «l'être vrai » d'une personne, sans que $\mathbf{S}$ puisse avoir accès à la réalité de la personne (c'est-à-dire $\mathbf{X}$ ) : le " personnage » construit s'oppose à la personne réelle, authentique. Bref, celui qui aime paraittre ne se laisse appréhender que par l'image qu'il donne de lui, comme l'énoncé suivant, extrait du corpus Frantext, le montre bien :

Mais cette fois, le geste était naturel. Il ne semblait dicté par aucun souci de paraître, de jouer, et ce fut juste quand Verdier évoqua cette rencontre de l'été, que le rapprochement devint inévitable. (M. Droit, Le Retour, 1964)

D'autre part, notre hypothèse permet de rendre compte de contraintes pesant sur les énoncés du type :

$$
\text { Son livre + son ouvrage }+ \text { la revue est paru(e). }
$$

Dans ces emplois, paraître est synonyme de publier, ce qui se comprend aisément, puisque ce dernier verbe signifie littéralement « rendre public $»^{23}$. On note cependant que, à la différence de publier, paraître exige, en position sujet, des lexèmes renvoyant à des objets ayant vocation à être diffusés : on fait paraître un livre, mais ?faire paraître un manuscrit est bizarre, alors qu'on peut très bien publier un manuscrit. De même, on fait paraittre un ouvrage, un article, mais on publie et non *fait paraître ses travaux ou ses recherches. On peut en outre faire paraître un traité (" ouvrage didactique »), une nouvelle ( roman court »), mais non *faire paraître un traité (« un accord entre pays »), ni une nouvelle (" un scoop»), alors que l'on peut publier ces derniers. Bref, dans ces

sur le comédien : «Réfléchissez un moment sur ce qu’on appelle au théâtre être vrai. Est-ce y montrer les choses comme elles sont en nature ? Aucunement. (...) Qu'est-ce donc que le vrai de la scène ? C'est la conformité des actions, des discours, de la figure, de la voix, du mouvement, du geste, avec un modèle idéal imaginé par le poète, et souvent exagéré par le comédien ».

23 Dans l'énoncé son disque est paru, paraître commute également avec sortir. Paraître est donc synonyme de deux verbes, sortir et entrer (cf. il parut + entra dans la pièce), qui sont eux-mêmes en relation d'antonymie. 
emplois, paraitre n'est compatible qu'avec des lexèmes renvoyant à des objets en euxmêmes destinés à un public.

\subsection{Description des énoncés du type Il ne laissa rien paraître de son malaise}

Il s'agira ici de vérifier que l'on retrouve les différents éléments du schéma métalinguistique formulé plus haut, dans les énoncés où paraitre est combiné avec un marqueur causatif, comme dans :

Suarès ne laisse que malgré lui paraître son ignorance. (A. Gide, Journal: 1889-1939, 1939)

On remarque tout d'abord que paraitre, dans ces énoncés, est associé à deux types de lexèmes: on a d'une part des mots renvoyant à un état interne d'un sujet, $a$ priori non partageable. Dans le corpus Frantext, les lexèmes de ce type les plus fréquents sont les suivants : douleur; détresse; lassitude; émotion; inquiétude; sentiments; soulagement; agacement; impatience; intérêt ; curiosité ; dépit; surprise ; déception; exaltation; trouble; crainte ; impressions ; embarras ; confusion ; dépression ; désir. On relève d'autre part une série de lexèmes qui s'interprètent comme des caractéristiques d'un sujet ou d'un objet, mais qui présentent la particularité d'être structurellement inaccessibles. Il peut s'agir d'un attribut d'un objet, comme dans :

Ce n'est pas que j'attachasse grande importance au costume, mais au contact de la grâce et de l'aisance de Sara, et par l'effet d'une extrême sympathie qui me fit voir avec ses yeux à elle notre intérieur, ce milieu dans lequel j'avais vécu jusqu'alors laissa paraître son insignifiance et sa conventionnelle banalité. (A. Gide, Geneviève ou la Confidence inachevée, 1936)

ou d'un sujet, comme dans :

M. De Charlus ne laissait pas seulement paraître une finesse de sentiment que montrent en effet rarement les hommes; sa voix elle-même (...) (M. Proust, La Recherche du temps perdu. À l'ombre des jeunes filles en fleur, 1918)

Dans tous les cas, le verbe paraitre n'est pas compatible avec des mots renvoyant à des entités possédant des caractéristiques directement perceptibles, alors que se voir ou être visible ne présentent pas cette contrainte. Ainsi dans l'énoncé suivant, paraitre est impossible :

Elle ne laissait rien voir (*paraître), du dehors, de ses cours intérieures et de ses jardins, mais Nicolas reconnut aussitôt la demeure qu'il n'avait jamais vue. (J. d'Ormesson, Le Vent du soir, 1985)

Par contre, on pourra avoir :

Si notre brusque arrivée l'avait troublée, du moins sut-elle n'en laisser rien voir (+ paraître).

(A. Gide, La Porte étroite, 1909)

Naturellement, j'en souffris beaucoup, mais voulus m'appliquer à n'en laisser rien voir (+ paraître).

(A. Maurois, Climats, 1928) 
De même, on peut laisser paraitre sa surprise, au sens de "étonnement", «stupeur», "stupéfaction», mais pas au sens de "mauvaise surprise», ou de «cadeau » (que l'on pourrait pourtant chercher à dissimuler) : il faut que la surprise soit éprouvée par quelqu'un. On peut aussi avoir Il fait paraitre la structure réelle de qch + la structure profonde de qch, mais non : *Il fait paraitre la structure d'accueil, alors qu'il est tout à fait possible de faire voir une structure d'accueil. De même, s'il est possible de faire paraitre sa joie + son allégresse + sa bonne bumeur + son entrain, ou sa gaieté, comme dans :

Les enfants eux-mêmes, qui avaient gồté le cachiri avant que fût tari le sein maternel, faisaient paraître une gaieté qu'ils semblaient ignorer jusqu'alors. (J. Perret, Roucou, 1936)

en revanche, on n'aura pas : *Les enfants faisaient paraitre une bilarité ..., parce que l'hilarité, contrairement à la joie, la bonne humeur, etc., est manifeste. Pour la même raison, on pourra avoir Il a laissé paraitre son amusement, mais non *Il a laissé paraitre son rire. Notons également la possibilité d'avoir :

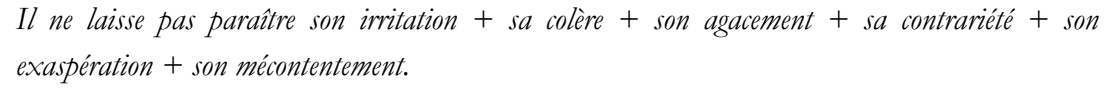

opposée à l'impossibilité d'avoir l'énoncé *Il ne laisse pas paraitre son emportement, emportement renvoyant à un comportement ostensible.

On a vu que le lexème en position de complément du groupe verbal laisser / faire paraître ne pouvait renvoyer à une entité directement perceptible. On l'assimilera donc, dans le schéma proposé plus haut, à l'élément $\mathbf{X}$, qui correspond à un élément en lui-même inaccessible.

Par ailleurs, la pertinence d'un paramètre $\mathbf{X}$ ' se manifeste, selon nous, dans le fait que l'on relève, dans ces énoncés, une problématique du déchiffrement, de l'interprétation de signes attestant de l'existence d'un état intérieur d'un personnage. Autrement dit, l'existence de $\mathbf{X}$ est révélée par la présence d'indices, de marques. Par exemple, l'énoncé suivant :

\section{(...) elle escamotait prestement la précédente, le tout d'une manière mécanique, l'air absent, ne laissant paraître ni agacement, ni impatience, ni intérêt, ni pour ce qu'elle faisait ni pour les acheteurs, pas plus que n'aurait pu manifester une machine à sous on un distributeur automatique. (Cl. Simon, L'Acacia, 1989)}

s'interprète comme «elle ne donnait aucun signe d'agacement, d'impatience » : ne rien laisser paraitre, est mis en parallèle, via la tournure participiale, à "avoir l'air absent», «agir de manière mécanique ». Les indices de $\mathbf{X}$ peuvent être inférés, mais ils sont souvent explicités dans le contexte immédiat, comme dans l'énoncé ci-dessous ${ }^{24}$ :

Mais, de nouveau maître de lui, il ne laissait paraître son émotion qu'à l'irrépressible titillement d'un petit muscle de sa joue. (A. Gide, Isabelle, 1911)

\footnotetext{
${ }^{24}$ Dans les exemples qui suivent, $\mathbf{X}$ figure en gras dans l'énoncé, et les indices ou signes de $\mathbf{X}$, c'està-dire $\mathbf{X}^{\prime}$, sont soulignés.
} 
François Thuillier

ou bien dans un contexte plus large, comme dans les deux énoncés suivants :

Sitôt donc que la porte se fut refermée sur ses gens, M. De Pinamonte laissa paraître tout le trouble dont il était agité. Baissant les yeux, se frottant rageusement les tempes, toussant, soufflant et maugréant dans le même temps, il m'entraîna dans la galerie. (O. Milosz, L'Amoureuse initiation, 1910)

Si le succès de la pièce justifiait cette sentence et surtout si le succès menaçait de s'éterniser, Éric Vidame laissait alors paraître une certaine inquiétude. Il disait à ses familiers : «la pièce est bonne, sans aucun doute, puisque nous l'avons jouée. C'est bon, mais n'exagérons pas \#.

(G. Duhamel, Chronique des Pasquier. Suzanne et les jeunes hommes, 1941)

La nature a priori cachée, intime, de $\mathbf{X}$, et l'existence d'indices à déchiffrer $\left(\mathbf{X}^{\prime}\right)$, explicités ou inférés, ont pour conséquence que les énoncés avec paraître renvoient fréquemment à un scénario de contrôle de son comportement. Il s'agit, pour le personnage, de ne pas se trahir par ses gestes, son attitude, ses paroles, son visage, bref de ne pas laisser voir de manifestations de son état intérieur, comme par exemple dans les énoncés suivants :

(...) rarement il avait vu des yeux aussi secs et aussi ardents tout ensemble, un homme aussi maître de ses nerfs, aussi capable de ne rien laisser paraître de ses sentiments. (G. Simenon, Les Vacances de Maigret)

Tour à tour, deux par deux, les femmes se levaient, les unes strictement voilées, les autres la figure découverte, et plus impénétrables encore tant elles mettaient d'application à ne rien laisser paraître de leurs sentiments sur leurs visages. (Jer. Tharaud, J. Tharaud, La Fête arabe, 1912)

La volonté de ne pas fournir d'indices de ce qui est ressenti peut déboucher sur une volonté de dissimulation, d'où une problématique de la duplicité, comme dans :

Seulement il semblait qu'un mur avait été dressé entre Lucie-maîtresse et Lucie-mère, car Sophie s'appliquait visiblement à ne rien laisser paraître $d u$ lien de parenté qui l'unissait à sa "maîtresse», et elle ne lui parlait qu'en l'appelant, comme nous autres, "Madame». (M. Tournier, Le Médianoche amoureux, 1989)

(...) surtout, je m'appliquais à ne rien laisser paraître de mes sentiments, à n'avoir l'air étonné de rien, choqué de rien. (G. Duhamel, La Confession de minuit, 1920)

On a vu que paraître renvoyait dans ces énoncés à la perception de signes $\left(\mathbf{X}^{\prime}\right)$ d'un $\mathbf{X}$ en lui-même hors d'atteinte. On peut opposer sur ce point paraître et apparaître, avec lequel la perception de la présence de $\mathbf{X}$ est de l'ordre du constat. Il est attaché aux énoncés comportant apparaître une nuance de clarté, d'évidence, d'univocité, qui leur est spécifique, comme en :

Ce fut, pour elle, ce moment d'angoisse où soudain l'adulte se trabit, laisse apparaître sa faiblesse, se sent un roi nu dans les yeux attentifs de l'enfant. (A. Makine, Le testament français, 1995)

Dans le même ordre d'idées, on notera que dans l'énoncé ci-dessous, $\mathbf{X}$ (la haine) s'interprète comme un stigmate : 
(...) et nous, nous pleurions de joie de voir la baine enfin apparaître sur ce corps, restreinte d'abord comme le bouton d'Alep, mais qui allait bientôt mordre et s'épanouir comme le cancer du soleil. (J. Giraudoux, Judith, 1931)

Avec apparaître, on peut interpréter « la haine » comme ce qui résulte de sévices, c'est-à-dire des marques laissées par la haine d'autres protagonistes. Si on remplace apparaître par paraître, la haine est celle qu'éprouve le personnage :

et nous, nous pleurions de joie de voir la baine enfin paraître sur ce corps, restreinte d'abord comme le bouton d'Alep, mais qui allait bientôt mordre et s'épanouir comme le cancer du soleil.

Avec paraître, la haine s'interprète donc non pas comme un stigmate, mais comme un symptôme. On notera d'ailleurs que, dans Frantext, dans tous les énoncés en faire/laisser paraître, combinés à un syntagme en par $Y, \mathbf{Y}$ s'interprète comme un révélateur de $\mathbf{X}$, comme dans :

C'était à qui témoignerait de plus grandes admirations après l'avoir oü. Qui les faisait paraître par des soupirs; qui, par une joie extraordinaire, laquelle se lisait sur les visages; qui, par un silence nécessaire et par une impuissance d'en dire ses sentiments. (H. Bremond, Histoire littéraire du sentiment religieux en France depuis la fin des guerres de religion jusqu'à nos jours, t. $3,1921)$

Le fait que $\mathbf{X}$ ne soit accessible que par l'intermédiaire de signes ou de marques explique aussi que, dans le corpus Frantext, la totalité des énoncés de la forme il n'y paraît (ra) plus renvoient à un effacement de traces, de séquelles. On peut toujours, même lorsque $\mathbf{X}$ ne figure pas explicitement dans l'énoncé, rétablir un événement détrimental, qui a occasionné une altération de l'état normal (maladie, désordre, etc.). Lorsque l'énoncé est au futur, la situation typique est celle d'un médecin ou d'un garde-malade, pronostiquant un retour à l'état normal (souhaité), comme dans :

À buit heures, Mme Petiot lui apporta une infusion. Elle lui tâta le pouls en disant:

- Ce ne sera rien. C'est demain mardi. Vous resterez. couché, et mercredi il n'y paraîtra plus.

(B. Clavel, La Maison des autres, 1962)

L'interprétation de ces énoncés, comme renvoyant à un effacement des traces, est spécifique des énoncés avec paraître. Dans les énoncés en ça ne se voit + verra plus, on interprète plutôt l'invisibilité comme une perte, comme dans :

Sans doute notre lampe faiblit, car, en dehors de ces choses proches, la magnificence du lieu ne se voit presque plus, s'indique plutôt à notre souvenir -par la silhouette rare de quelque vase de cinq cents ans, par le reflet de quelque inimitable soierie (...) (P. Loti, Les Derniers jours de Pékin, 1902)

On a essayé de montrer que les énoncés en laisser / faire paraître renvoyaient à une problématique de l'appréhension de quelque chose grâce à des symptômes, ou des signes de ce quelque chose, qui sont perçus par un sujet. En d'autres termes, ces énoncés renvoient à un processus de perception de signes $\left(\mathbf{X}^{\prime}\right)$ de $\mathbf{X}$, c'est-à-dire 
d'entités secondes par rapport à $\mathbf{X}, \mathbf{X}$ étant en lui-même inaccessible $\mathbf{X}$ est de l'ordre de l'intime, ou correspond à une caractéristique invisible ${ }^{25}$.

\subsection{Description des énoncés du type Cette longue journée parut lente au jeune homme}

On propose maintenant d'analyser les emplois où, pour reprendre la terminologie traditionnelle, paraitre correspond à un auxiliaire de modalité épistémique, les énoncés exprimant un jugement sur la vérité de l'énoncé, ou sur la réalité d'un état de choses. Notre objectif, dans cette section, sera d'une part d'identifier plus précisément la valeur des emplois modaux de paraitre, à l'aide d'une comparaison avec un proche synonyme, le verbe sembler, et d'autre part de montrer que les effets de sens spécifiques de paraitre dans ces emplois sont cohérents avec les caractéristiques des énoncés que l'on a décrits dans les deux premières sections. Il faudra enfin rendre compte de la valeur globale des énoncés, c'est-à-dire rendre compte de la modalisation qu'induit paraitre, en fonction du schéma proposé plus haut.

On trouve, dans la littérature consacrée à paraître et sembler, peu d'indications précises sur la valeur des énoncés comportant paraitre, qui sont le plus souvent définis de manière circulaire, en termes d'« apparence $»^{26}$. On va s'attacher à montrer que la spécificité interprétative des énoncés avec paraître est qu'ils renvoient à une explicitation des impressions, des sensations, a priori irréductibles et indicibles d'un sujet, alors que les énoncés avec sembler correspondent à des conjectures, des hypothèses. On peut dans un premier temps illustrer ceci à l'aide d'une opposition telle que :

Elle paraît plus jeune que sa voisine.
Elle semble plus jeune que sa voisine.

Avec paraitre, il s'agit de transcrire l'impression qu'elle donne, l'effet qu'elle produit, alors qu'avec sembler, c'est une conjecture. Le second énoncé signifie « je pose qu'elle est plus jeune que sa voisine, mais je ne suis pas sûr ». On notera à ce propos que si on peut avoir :

Même il paraissait plus jeune encore que la première fois que l'avait rencontré Julius. (A. Gide, Les Caves du Vatican, 1914)

\footnotetext{
${ }^{25}$ Selon le Petit Robert, un signe est une "chose perçue qui permet de conclure à l'existence ou à la vérité d'une autre chose, à laquelle elle est liée".

${ }^{26}$ Cf. par exemple, M. Riegel et al. (1994 : 237). Cf. également P. Bourdin (1986 : 46), pour qui paraître et sembler sont «le support d'un jugement d'apparence, opération de nature épistémique qui incorpore, sans y être réductible, un jugement de probabilité ». P. Bourdin fournit plus loin dans son étude contrastive de paraître et sembler une hypothèse concernant la valeur des deux verbes, que l'on ne discutera pas ici faute de place. Pour un compte-rendu de l'article de P. Bourdin, et pour une analyse plus détaillée de la littérature consacrée à paraître dans ces emplois, nous renvoyons à $\mathrm{F}$. Thuillier (à paraître).
} 
en revanche, il serait peu plausible de rencontrer un tel énoncé avec le verbe sembler, puisqu'on peut difficilement faire l'hypothèse que quelqu'un est plus jeune qu'à un autre moment, si ce moment est antérieur. Avec paraitre, il n'y a pas de contradiction, parce que l'on parle des impressions, de l'effet que fait quelqu'un sur un sujet. Une des particularités de paraitre est ainsi de permettre une autonomie des perceptions d'un sujet par rapport à la réalité, les impressions d'un sujet n'ayant pas nécessairement à être conformes à la réalité pour exister. Un locuteur peut ainsi dire, sans contradiction, que quelque chose a telle qualité, et prédiquer une qualité contraire, comme dans l'énoncé ci-dessous, où l'on oppose la représentation d'un sujet (impression de précision, due à l'ivresse) et ce que le sujet sait par ailleurs être réel :

\footnotetext{
"Demain, oui pas plus tard que demain, je vais téléphoner à Ben. Il sera mon Patricia, Ben, et il me guérira de Patricia. » Un défilé de phrases floues qui me paraissaient bougrement précises. Rien de tel que les boissons alcoolisées pour vous donner l'impression que l'on frôle la vérité et que l'on a du génie. (B. Groult, Fl. Groult, Il était deux fois, 1968)
}

Cette différence entre une explicitation des impressions d'un sujet avec paraitre, et l'expression d'une conjecture ou d'une hypothèse avec sembler, se révèle également dans des énoncés tels que :

\section{Dans ce pays, l'eau paraissait plus vive et plus puissante que partout ailleurs. \\ Comme les chats, elle se lave peu, mais elle paraît toujours propre.}

qui renvoient à des sensations, dont la véracité n'est pas pertinente, et où sembler pourrait difficilement être substitué à paraître.

Comme on l'a vu, la valeur des énoncés comportant paraitre peut être décrite en termes d'impressions d'un sujet, ou d'effet produit par quelque chose sur un sujet. On retrouve ici les caractéristiques des $\mathbf{X}$ ' que l'on avait mises au jour pour les autres emplois, puisque ce qui est perçu correspond à une entité seconde par rapport à un $\mathbf{X}$ : une impression est en effet une marque, trace, laissée par quelque chose sur une autre chose $^{27}$.

L'hypothèse selon laquelle paraître renvoie régulièrement à une mise en relation d'un $\mathbf{X}$ en lui-même hors d'atteinte et un $\mathbf{X}^{\prime}$ perçu, second par rapport à $\mathbf{X}$, permet de rendre compte de la modalisation associée à paraitre : les énoncés renvoient à une mise en relation entre une qualité prédiquée d'un objet de manière absolue, que l'on pourrait noter à l'aide de la copule être, et une impression. Dans Ça (me) paraît bon, on a une mise en relation entre un $\mathbf{X}$ " c'est bon », posé dans l'absolu (ce que c'est), et un $\mathbf{X}^{\prime}$, qui correspond à une impression de «c'est bon » perçue par un sujet. On obtient ainsi une relativisation de la prédication, puisque paraitre marque qu'une chose ne peut être appréhendée que par l'impression qu'elle procure. Selon cette hypothèse, il demeure toujours, avec paraitre, un écart entre ce qu'est une chose (en soi inaccessible)

\footnotetext{
${ }^{27}$ Dans son acception technique, l'impression correspond à un procédé de reproduction (cf. la recréation d'un monde fictif sur une scène dans les emplois intransitifs décrits plus haut), quelque chose gardant l'empreinte d'une autre chose.
} 
et l'impression qu'elle produit; on peut noter à ce propos que le fait de poser explicitement $\mathbf{X}$ dans l'énoncé, à la forme négative, comme par exemple dans :

Il n'est pas aussi idiot qu'il le paraît.

a pour effet de souligner l'écart irréductible qui existe entre $\mathbf{X}$ et $\mathbf{X}$ ', d'où une interprétation globale de discordance entre "ce qui est" et l'impression perçue. La distance entre $\mathbf{X}$ et $\mathbf{X}$ ' peut donc faire émerger une problématique de l'illusion, du caractère trompeur de $\mathbf{X}$ ', ce que l'on peut rapprocher de la dissimulation des sentiments que l'on observait dans les emplois du type il ne laissa rien paraitre de sa joie, et de la duplicité de celui qui aime paraitre : à chaque fois, c'est l'écart entre $\mathbf{X}$ et $\mathbf{X}^{\prime}$ qui autorise l'apparition de ce type d'interprétations.

\subsection{Description des énoncés du type Il paraît qu'il a perdu une grosse somme au jeu}

On conclura en vérifiant que le schéma proposé en 2. permet de rendre compte de la valeur des emplois dits " évidentiels », ou « médiatifs » de paraitre ${ }^{28}$, dont la valeur est traditionnellement décrite en termes de « rumeur», de «bruit qui court», ou de « ouï-dire ».

Ces énoncés renvoient à la transmission d'une information à un public (noté $\mathbf{S}$ ), et indiquent qu'il s'agit nécessairement d'une information de seconde main: le locuteur d'un énoncé en il paraît que ne peut, en effet, avoir été le témoin direct de l'événement qu'il relate, d'où le fait que ce locuteur soit posé comme ne garantissant pas la véracité de l'information qu'il transmet.

En d'autres termes, on a affaire, dans ces énoncés, à un événement en luimême inaccessible (que l'on note donc $\mathbf{X}$ ), mais qui se trouve appréhendé grâce à un récit de cet événement (c'est-à-dire par le biais d'une entité seconde par rapport à $\mathbf{X}$, notée $\mathbf{X}^{\prime}$ ). On retrouve donc un $\mathbf{X}$ qui reste hors d'atteinte, mais qui se trouve appréhendé par $\mathbf{S}$ par le biais d'un $\mathbf{X}$ ', second par rapport à $\mathbf{X}$.

\footnotetext{
${ }^{28}$ Selon P. Dendale et L. Tasmowski (1994: 5), «un marqueur évidentiel est une expression langagière qui apparaît dans l'énoncé et qui indique si l'information transmise dans cet énoncé a été empruntée par le locuteur à autrui, ou si elle a été créée par le locuteur lui-même, moyennant une inférence ou une perception ».
} 
Le verbe paraittre : surgissement, manifestation, impression, oü-dire

\section{RÉFÉRENCES BIBLIOGRAPHIQUES}

BOURDIN, P., 1986, «Sembler et paraître, ou les deux visages de l'apparence », Semantikos, vol. 10, fasc. 1-2. Paris : Semantikos, 45-67.

DALADIER, A., 1990, «Aspects constructifs des grammaires de Zellig Harris », Langages, 99. Paris : Larousse, 57-84.

DENDALE, P., TASMOWSKI, L., 2001, «Evidentiality», Journal of Pragmatics: An Interdisciplinary Journal of Language Studies, 33, 3. Amsterdam: Elsevier, 339-464.

DUPAS, Ch., 1997, Perception et langage : étude linguistique du fonctionnement des verbes de perception auditive et visuelle en anglais et en francais. Louvain - Paris : Peeters.

FILLMORE, C. J., 1970, «Verbes de jugement », Langage, 17. Paris : Larousse, 56-72.

GALLEPE, T., 1997, Didascalies. Les mots de la mise en scène. Paris : L'Harmattan.

GAYRAL, F., 1998, "Créativité du sens en contexte et hypothèse de compositionnalité », TAL, vol. 39, 1. Paris : Atala ; Saint-Cloud : TAL, 67-98.

GLEASON, H. A., 1961, An Introduction to Descriptive Linguistics. New-York : Holt, Rinehart and Winston.

GUENTCHEVA, Z., 1994, "Manifestations de la catégorie du médiatif dans les temps du français », Langue Française, 102. Paris : Larousse, 8-23.

GUIMIER, C., 1991, "Sur la fonction "attribut du sujet" : approche synchronique » in À la recherche de l'attribut, éd. par M.-M. DE GAULMYN, S. RÉMI-GIRAUD. Lyon : Presses Universitaires de Lyon, 209-235.

OUELLET, P., 1992, "Quantité et qualité dans la représentation de la perception » in La Quantité et ses modulations qualitatives, éd par J. FONTANILLE. Limoges : PULIM, 175197.

OUELLET, P., EL ZAÏM, A., BOUCHARD, H., 1994, «La représentation des actes de perception : le cas de paraître », Cabiers de Praxématique, 22. Montpellier : Université Paul Valéry, 135-156.

POIRIER, C., MATHET, Y., ENJALBERT, P., 1998, «La compositionnalité à l'épreuve des faits, à travers un projet de compréhension automatique de constats d'accidents », TAL, vol. 39, 1, Paris : Atala ; Saint-Cloud : TAL, 99-129.

RIEGEL, M. et al., 1994, Grammaire méthodique du français. Paris : Presses Universitaires de France.

THUILLIER, F., 2003, Systématique des emplois du verbe paraittre en français contemporain. Thèse de doctorat, Université de Paris 7.

THUILLIER, F., à paraître, "Synonymie et différences : le cas de paraître et sembler», Enjeux, Revue du Centre d'Etudes et de Documentation pour l'Enseignement du Français. Namur : Facultés Universitaires Cedocef.

ÜBERSFELD, A., 1982, Lire le théatre. Paris : Editions sociales. 
François Thuillier

VANDELOISE, Cl., 1986, L'espace en français : sémantique des prépositions spatiales. Paris : Editions du Seuil.

VANDELOISE, Cl., 1990, "Les frontières entre les prépositions sur et à», Cabiers de Grammaire, 15. Toulouse : Université de Toulouse-le-Mirail, 157-184. 